\title{
Percepción de las motivaciones en el ingreso a una carrera del área de la salud
}

\author{
Claudia Troncoso Pantoja ${ }^{1}$, Bárbara Garay Lara ${ }^{2}$, Paulina Sanhueza Muñoz ${ }^{2}$
}

\section{RESUMEN}

Objetivo: Interpretar la percepción de las motivaciones que presentan estudiantes a ingresar en una carrera del área de la salud en la Facultad de Medicina de la Universidad Católica de la Santísima Concepción, Chile durante el año 2015.

Material y Métodos: A través de un diseño cualitativo y utilizando un enfoque fenomenológico, se aplica entrevista semiestructurada a 55 estudiantes que ingresan a la carrera de Medicina, Enfermería, Nutrición y Dietética, Kinesiología o Tecnología Médica de la Facultad de Medicina de la Universidad Católica de la Santísima Concepción, Chile, durante el año 2015. Los resultados, sometidos a triangulación de informantes, son analizados utilizando la técnica de análisis de discurso.

Resultados: La vocación de servicio público, el campo laboral una vez egresados o la vivencia de experiencias personales y/o familiares frente a una condición de salud se perciben como parte importante en las motivaciones de ingreso a una carrera del área de salud. La familia, el establecimiento educacional de procedencia o los amigos no se identifican como influyentes en la selección de la carrera de formación profesional a seguir. Esta situación es identificada independiente de la disciplina seleccionada.

Conclusiones: El altruismo y la vocación de servicio público son percibidos como el principal factor condicionante al momento de ingresar a una carrera del área de salud.

Palabras Claves: Motivación; estudiantes de área de la salud; análisis cualitativo.

\section{Perception of the motivational factors for pursuing a career in healthcare}

\section{ABSTRACT}

Objective: To interpret the perception of the motivational factors presented by students who decide to pursue a career in healthcare in the Faculty of Medicine at the Catholic University of the Most Holy Conception, Chile during 2015.

Material and Methods: Through a qualitative design and using a phenomenological approach, a semi-structured interview was applied to 55 new students. They were all pursuing a degree in the fields of Medicine, Nursing, Nutrition and Dietetics, Physical Therapy or Medical Technology of the School of Medicine at Universidad Católica de la Santísima Concepción, Chile, in 2015. The results were subject to triangulation of informants, and were analyzed using the technique of discourse analysis.

Results: Vocation for public service, the career field once graduated or the experience of personal /familiar experiences when facing a health condition are perceived as an important part of the motivational factors for pursuing a degree in healthcare. Family, school where they graduated from or friends are not identified as an influence when deciding what professional field to pursue. This situation is not necessarily linked to a specific area of expertise.

Conclusions: Altruism and vocation for public service are perceived as the main conditioning factor when deciding to pursue a degree in healthcare.

Keywords: Motivation; Healthcare students; Qualitative analysis.

\footnotetext{
${ }^{1}$ Magister en Salud Pública Basada en Evidencias. Facultad de Medicina. Departamento de Ciencias Clínicas y Preclínicas. Carrera de Nutrición y Dietética. Universidad Católica de la Santísima Concepción. Chile.

${ }^{2}$ Licenciada en Nutrición y Dietética. Facultad de Medicina. Carrera de Nutrición y Dietética. Universidad Católica de la Santísima Concepción. Chile.
} 


\section{INTRODUCCIÓN}

Independiente de la labor que desarrolla una persona, debe existir un motivo para su realización. Motivación puede ser definida como la actitud que presentan las personas al ejecutar una actividad, permitiendo direccionar su comportamiento, a través de un proceso dinámico interno que puede dar respuesta a la variabilidad en el comportamiento humano (1-2).

La presencia de motivación para el desarrollo de actividades laborales constituye un comportamiento adaptativo con el que se responde en forma eficaz a las demandas que se presentan en el trabajo. Un buen ambiente laboral necesita de esfuerzo social y de recursos para el logro de metas individuales y colectivas (3-5).

En el ámbito de la salud, se identifican como factores que condicionan la motivación en el desempeño y compromiso laboral de los agentes sanitarios a la vocación de servicio que presente el profesional; el nivel de remuneraciones obtenido; el clima laboral/organizacional y la capacidad de crecer profesionalmente en el lugar en donde desempeñe sus funciones disciplinares (6-8). Sin embargo, se reconocen como aspectos negativos en la vida laboral en esta misma área a la carga de trabajo que presentan los funcionarios; el limitado apoyo directivo y por sobre todo, la falta de motivación en el trabajo (9-11).

Si bien los fundamentos para desarrollar un trabajo provienen de motivaciones personales, hay diversas situaciones que podrían fortalecer esta instancia, como lo es la etapa de formación disciplinar en la educación superior. La motivación que presentan estudiantes universitarios al ingresar a la universidad se centran especialmente en el aprendizaje, pero también su ingreso está favorecido por la vocación, la admiración a los profesionales de área que les interesa, la entrega de valores de la respectiva disciplina o factores sociales en donde resalta especialmente el grupo familiar (12-16).

Es importante destacar que se espera de un estudiante que ha seleccionado para su formación profesional una carrera del área de salud, que no solo esté motivado en estudiar una determinada disciplina, sino que además sienta la vocación por formarse en esta última. La motivación se relaciona a los gustos o preferencias que la persona quiere desarrollar bajo diversas circunstancias, en cambio la vocación se entiende como el llamado o la necesidad imperiosa de cumplir con el destino. Es por esto que en el trascurso de la vida, se puede estar motivado a realizar una determinada acción sin presentar necesariamente vocación al ser desarrollada. La vocación en carreras del área médica depende del interés de casa individuo, complementado por un ambiente social y familiar que fortalecen y estimulan su desarrollo, con el fin de lograr una actitud positiva para la atención a la población que necesita de sus servicios (17-21).

\section{MATERIAL Y MÉTODOS}

Para el logro de los objetivos de este estudio, se utilizó el paradigma cualitativo con enfoque fenomenológico, lo que permite profundizar en el motivo de estudio desde el medio en donde ocurre (22). Este último corresponde a estudiantes que ingresan a una de las carreras que dicta la Facultad de Medicina de la Universidad Católica de la Santísima Concepción, Concepción, Chile durante el año 2015.

Para ingresar a esta investigación y como parte de sus criterios de selección, los alumnos debían pertenecer a una de las carreras que imparte la Facultad de Medicina de la Casa de Estudios antes mencionada: Medicina, Enfermería, Nutrición y Dietética, Kinesiología o Tecnología Médica, ingresando en marzo del año 2015 y firmando el consentimiento informado para este estudio, en donde se le indicaba sobre los objetivos de la investigación, la metodología de recolección de la información y la garantía de la privacidad de sus antecedentes personales a cada uno de los entrevistados. Esto último fue parte de las consideraciones éticas en la investigación.

La recolección de la información se realizó utilizando entrevistas semiestructuradas, validadas por expertos y sometida a prueba piloto. Las preguntas se basaban en la profundización de las influencias que ejercen el entorno social (familia, amigos y establecimiento educacional) y la presencia de vocación de servicio o experiencias personales que favorecen la elección de una carrera del área médica. Esto último es de importancia en la investigación, ya que se pretende identificar si los estudiantes novatos solo están motivados o realmente presentan vocación en la elección de su futuro profesional. 
Las entrevistas fueron realizadas por dos de los investigadores principales, en condiciones estándar en dependencias de la Facultad de Medicina. Estas fueron grabadas y traspasadas a formato papel para ser analizadas mediante el "análisis de discurso", que permite indagar en la lengua oral o escrita, más allá de los límites de la oración y de las propiedades de la comunicación diaria (23). Para fortalecer la validez y confiabilidad de los resultados de la profundización del discurso, se utilizó como estrategia la "triangulación de investigadores", técnica que recurre a más de un investigador para recolectar y analizar los datos (24). En este estudio, dos de los investigadores analizaron en forma independiente los discursos entregados por los entrevistados para posteriormente comparar los resultados.

El muestreo fue de tipo no probabilístico por conveniencia, acorde a los criterios de selección del estudio. Para el tamaño muestral, se utilizó el "punto de saturación teórico" esto es, las respuestas entregadas por los entrevistados no aportan una nueva información que sea relevante para los objetivos del estudio (25). En esta investigación la saturación se obtuvo para la carrera de Medicina en 7 entrevistas; para Enfermería en 13 entrevistas; Nutrición y Dietética en 10 entrevistas; Kinesiología en 16 entrevistas y para Tecnología Médica en 9 entrevistas. En total, se realizaron 55 entrevistas en estudiantes que presentaban la siguiente caracterización: 36 eran de sexo femenino (65.5\%) y 19 (34.5\%) de sexo masculino. La edad promedio de los estudiantes era de 18.2 años (+/- 1.18 años), con un rango entre 17 a 31 años.

\section{RESULTADOS}

\section{Entorno social}

\section{Familia}

La familia, como parte esencial del entorno social y en especial los padres, son identificados por gran parte de los participantes del estudio como un factor que no motiva la elección de su carrera universitaria, debido a que su opción académica se debe más a un interés personal que a una decisión de tipo familiar. Esta situación se presenta especialmente en las carreras de Enfermería, Nutrición y Dietética, Kinesiología y Tecnología Médica. Los estudiantes de Medicina, por el contrario, en su mayoría opinan que sintieron la influencia especialmente parental y que los consejos de la familia intervinieron en su ingreso universitario.
Los padres en especial, son mencionados por una minoría de los entrevistados, identificando que de cierta manera, condicionan su selección a través de la percepción que tienen estos últimos de las carreras del área médica, con opiniones positivas relacionadas al asegurarse un futuro social y económico o con percepciones negativas relacionadas a la mantención de una cultura familiar de ciertas profesiones de otras áreas profesionales.

Sin bien los estudiantes no condicionan su elección universitaria a la opinión de sus familiares, identifican en su mayoría que una vez seleccionados en sus respectivas carreras, sus familiares apoyan la decisión tomada.

\section{Amigos}

Al igual que lo que ocurre con el círculo familiar, gran parte de los entrevistados identifican que sus amigos, en especial sus pares en el colegio, no interfieren en su motivación a ingresar a una carrera del área médica.

Para algunos de los estudiantes, los amigos cercanos tenían opciones similares a los personales, por lo que no condicionaron su decisión. Por el contrario, algunos de los entrevistados identifican que algunos de sus amigos de la educación secundaria motivaban el seguir carreras del área humanista o alguna ingeniería, para mantener su amistad en las aulas durante la educación superior.

Una minoría, en especial de la carrera de Medicina, menciona que eran reservados en su opción académica. Esto sería por la dificultad que conlleva el ser aceptado en esta carrera.

Al igual que lo que ocurre con sus familias, una vez seleccionado en sus respectivas carreras, la mayoría de los entrevistados identifican el apoyo que sus amigos le brindan a su opción universitaria.

\section{Establecimiento educacional}

La mayoría de los estudiantes de las carreras de Kinesiología, Nutrición y Dietética y Enfermería entrevistados identifican la limitada o nula orientación recibida desde los establecimientos educacionales para la selección de su carrera universitaria y si esta se presentó, no fue significativa para definir su futuro profesional. 
En cambio, gran parte de los estudiantes que ingresaron a la carrera de Medicina y Tecnología Médica identifican un apoyo vocacional por parte de sus colegios o liceos, los que les permitió elegir su carrera en forma consciente e informada.

Un número limitado de los entrevistados refiere haber asistido a los programas "Casa Abierta" que mantienen las Universidades en la región (instancia en que las Casas de Estudio Superior reciben a estudiantes secundarios y dan a conocer sus actividades de extensión y académicas de las diversas carreras que imparten las Instituciones), lo que fue percibido como un factor positivo al momento de definir la carrera a estudiar.

\section{Vocación de Servicio}

A excepción de los estudiantes que ingresaron a la carrera de Nutrición y Dietética, gran parte de los entrevistados identifican que sentían desde su infancia el querer estudiar una carrera del área de la salud. Esto último lo codifican como "vocación de servicio": el contacto y el querer ayudar a la persona necesitada, vulnerable o con alguna patología asociada. Identifican la necesidad de empatía e interacción con el paciente para el ejercicio de la profesión que han escogido y por sobre todo, el servicio social que ejercerán con su futura profesión.

En cambio, los estudiantes que ingresaron a la carrera de Nutrición y Dietética opinan que su primer acercamiento para desarrollar una carrera de servicio en el área médica surge en su adolescencia.

Algunos estudiantes de la carrera de Medicina identifican las carencias en la salud pública que presenta actualmente el país y que afecta a la población más vulnerable de la sociedad chilena. Ellos declaran que una de las motivaciones a ingresar a esta carrera es el aporte que ellos perciben podrán brindar a la sociedad para paliar esta situación en salud.

Estudiantes de Enfermería, Kinesiología y Nutrición y Dietética identifican el servicio que realizarán en el ejercicio de su profesión antes de conocer en profundidad su carrera: conseguir una alimentación sana o terapias de rehabilitación, por ejemplo, con el fin de lograr el bienestar en salud.

\section{Experiencias personales}

La experiencia personal en situaciones de alteración del estado de salud o de presentar familiares cercanos con algún tipo de enfermedad, junto a la necesidad de ayudar y su falta de herramientas para colaborar en este propósito, son esenciales en la motivación de la selección de la carrera profesional de un número importante de estudiantes de Enfermería, Kinesiología, Nutrición y Dietética y Tecnología Médica.

El campo laboral que presenta la disciplina es mencionado constantemente por algunos entrevistados de la carrera de Tecnología Médica, Kinesiología y Nutrición y Dietética, ya que el nivel de ocupación una vez egresados es percibido por estos estudiantes como un factor importante al seleccionar esta carrera.

Un número importante de estudiantes de Medicina y una minoría de la carrera de Enfermería y Nutrición y Dietética, identifican como parte de su vivencia personal, la situación actual de la salud pública en Chile en relación a la cantidad de médicos que trabajan en esta área, codificando la vocación de servicio público como parte de su motivación al ingreso a estas carreras.

Una minoría de los entrevistados se sentía motivada a ver el trabajo que realizaban médicos o enfermeros en centros de salud, lo que es percibido por ellos como un factor importante al momento de elegir una carrera del área de salud, ya que se proyectaron realizando este trabajo de servicio a la comunidad en la práctica clínica.

Siendo una de las bases en la selección de la profesión del área médica la vocación de servicio, los aspectos económicos fueron considerados por un número muy reducido de estudiantes de las carreras de Medicina y Enfermería, opinando que las remuneraciones en esta área son adecuadas para un buen vivir. Esta situación se invierte para los estudiantes de Tecnología Médica y Nutrición y Dietética, en que un número importante de entrevistados consideraron los aspectos económicos que lograrán al egresar de la disciplina seleccionada. 


\section{DISCUSIÓN}

Se encuentra en la literatura científica diversas investigaciones que identifican las motivaciones que los estudiantes presentan para seguir una carrera universitaria como son la iniciativa propia, el conseguir un título profesional, el reconocimiento y éxito personal, la vocación, entre otras (26-30).

Se esperaría que los futuros profesionales de carreras del área médica presenten además, una la visión de servicio a las necesidades de las personas que serán atendidas por ellos una vez que concluyan su formación profesional.

En nuestro estudio, realizado en alumnos de las carreras del área de la salud que oferta la Facultad de Medicina de la Universidad Católica de la Santísima Concepción, Chile, durante el año 2015, las motivaciones encontradas para la selección de su futura profesión se centran especialmente en las aspiraciones altruistas de los estudiantes, identificando la vocación de servicio público para contribuir a la sociedad, como una de las bases en la selección de la carrera escogida. Esta situación se replica en estudios, como los realizados por Díaz et al. y Machado et al (31-32). Se ha identificado la importancia que presenta para los componentes del cuerpo asistencial de una determinada institución de salud, el presentar la "vocación de servicio", esto debido al medio en donde realizarán su quehacer profesional y el carácter solidario y humanista de sus respectivas disciplinas (33-34). Los estudiantes que participaron en el estudio, en su mayoría, identifica su capacidad de servicio, lo que ya es un paso en obtener una buena atención al usuario por parte de estos futuros profesionales y refuerza el hecho que los formadores en esta institución de Educación Superior, deben procurar fortalecer esta cualidad en sus alumnos.

En cuanto al entorno social, referido a la familia y amigos, los estudiantes entrevistados no identifican como un factor motivacional desencadenante en la elección de su futura profesión. Esta limitada significación es compartida en estudios realizados por Peinado et al y De la Mano et al (35-36). Si bien, los alumnos identifican los consejos que en especial los padres les entregan antes de postular a las carreras de su preferencia, estas recomendaciones no son condicionantes en su decisión final.
El establecimiento educacional en la adolescencia es percibido por gran parte de los estudiantes de la carrera de Medicina como un factor que favoreció su elección, ya que identificaron que la orientación que recibieron en la educación secundaria actuó como reforzamiento positivo a su elección académica, al igual que los resultados encontrados por Mejías et al (37). Sin embargo, la gran parte de los entrevistados de las otras carreras de la Facultad de Medicina, considera que no recibieron orientación suficiente en sus respectivos liceos o colegios para motivar la elección de su carrera universitaria, como los resultados encontrados por Fé de la Mella (38). También existe un grupo de estudiantes entrevistados que perciben cierta influencia vocacional por parte de sus respectivos centros de educación secundaria, esta no fue lo suficientemente motivador como para condicionar su elección profesional.

La situación del sistema de salud en el país también es considerada por un segmento de los participantes de la investigación como condicionante en su elección profesional. En Chile, en los últimos años existe una opinión desfavorable por parte de los usuarios externos de sus servicios, originado por factores en los que se destacan las listas de espera a diversos servicios o el acceso a especialidades médicas, entre otros (39). Algunos de los entrevistados identifican la necesidad de mejorar esta condición a través del apoyo que brindarán una vez egresados, como parte del cuerpo de salud. Esta situación se percibe de mayor manera en estudiantes de la carrera de Medicina.

Otras condicionantes en la percepción de las motivaciones a ingresar a una carrera del área de salud encontradas en esta investigación y que concuerda con otros estudios se refiere a mantener la calidad de vida de los entrevistados (40); la demanda laboral que presenta la respectiva disciplina seleccionada y que asegurarán, desde su punto de vista, una rápida y adecuada inserción laboral (41) o el cumplimiento de expectativas personales $y$ económicas (42-44).

En conclusión, los estudiantes están motivados en estudiar una carrera del área médica y que la vocación o las aspiraciones altruistas predominan en los estudiantes que ingresan a una carrera del área de salud en la Universidad Católica de la Santísima Concepción durante el año 2015. La vocación de servicio público es percibida como factor 
Percepción de las motivaciones en el ingreso

a una carrera del área de la salud

condicionante en la mayoría de los estudiantes entrevistados. La familia, amigos y el establecimiento educacional no presentan una influencia significativa en la selección de la carrera profesional de estos jóvenes, que inician su vida universitaria.

Es necesario e importante considerar que los futuros agentes de salud, deben presentar e identificar una inspiración marcada hacia el bien común, con aspiraciones de servicio a mejorar la calidad de vida de las personas atendidas. Si estas actitudes no están presentes desde los inicio de formación profesional, tendremos funcionarios preocupados por un número de ficha clínica o de una cama, sin considerar las necesidades que presenta la persona detrás del individuo.

\section{REFERENCIAS BIBLIOGRÁFICAS}

1. Figueroa E, Mensaque J et al. Jujuy. Terciario. Motivación. Cuad. Fac. Humanid. Cienc. Soc., Unid. Nac. Jujuy. 2009; 37:279-289.

2. Martinez M, Alba L et al. Interrelación dialéctica entre calidad de vida y motivaciones relativas a la salud. 2010; 26(1): 0-0.

3. Navarro J, Ceja L, Curioso F et al. Cómo motivar y motivarse en tiempos de crisis. Papeles de Psicólogo. 2014; 35(1): 31-39.

4. Segredo A. Caracterización del sistema de dirección de la atención primaria de salud. Rev. Cubana de Salud Pública 2009; 35(4): 78-109.

5. Feldman L, Avellán M, Lugli Z. Percepción del ambiente laboral y variables personales en gerentes venezolanos. Anales de la Universidad Metropolitana. 2012; 12(2): 145-166.

6. Vieira A, Vieira M et al. Factores de motivación e insatisfacción en el trabajo del enfermero. Rev. esc. enferm. USP. 2005; 39(1):85-91.

7. Bernal I, Pedraza $\mathrm{N}$ et al. El clima organizacional y su relación con la calidad de los servicios públicos de salud: diseño de un modelo teórico. estud. gerenc. 2015; 31(enero-marzo):20-29.

8. Muñoz E, Coll J et al. Influencia del clima laboral en la satisfacción de los profesionales sanitarios. Aten Primaria 2006; 37(4): 209-214.

9. Hanzeliková A, García M et al. La calidad de vida profesional en las enfermeras de geriatría. Enferm.glob.2011;10(24): 0-0.

10. Zubiri F. Satisfacción y Motivación Profesional. An. Sist. Sanit. Navar. 2013; 36(2):193-196.

11. Pérez $\mathrm{L}$, Soler $\mathrm{S}$ et al. Ambiente laboral en los policlínicos universitarios. Educ Med Super 2009; 23(2): 0-0.

12. Míguez $M$, Crisci $C$ et al. Herramienta diagnóstica al ingreso a Facultad de Ingeniería: motivación, estratégias de aprendizaje y conocimientos disciplinares. Revista Argentina de Enseñanza de la Ingeniería 2007; 8(14):29-37.
13. Mas C, Medinas M. Motivaciones para el estudio en Universitarios. Anales de psicología. 2007; 23(1):17-24.

14. Ortiz M. Principales señas de identidad de los estudiantes de comunicación en el EES de la comunidad de Madrid en 2012: expectativas, perfil de opción y relaciones mediáticas. Estudios sobre el mensaje periodístico 2012; 18(noviembre): 661-670.

15. Carrasco E, Osses S. Transformaciones del perfil valórico en estudiantes de la carrera de obstetricia y puericultura de la Facultad de Medicina de la Universidad de la Frontera durante su trayectoria académica. Estud. pedagóg. 2008; 34(2): 45-63.

16. Donoso S, Schiefelbein E. Análisis de los modelos explicativos de la retención de estudiantes en la Universidad: una visión desde la desigualdad social. Estud. pedagóg. 2007; 33(1): 727.

17. Piña J. Motivación en psicología y Salud: motivación no es sinónimo de intención, actitud o percepción de riesgo. Revista Diversitas - perspectivas en Psicología. 2009; 5(1): 27-35.

18. Mazo H. Vocación por el hacer y el ser en las profesiones del sector salud. Revista Humanismo Soc. 2014; 2(2): 52-64.

19. Perales A, Sánchez E, Mendoza A et al. Vocación médica en estudiantes de medicina de un universidad peruana. An. Fac. med. 2014; 75(1): 37-42.

20. Perales A, Mendoza A, Sánchez E. Vocación médica en médicos de prestigiada conducta profesional. An. Fac. med. 2013; 74(4): 291-300.

21. González R. Humanismo y gestión de salud: pasado, presente y futuro. Rev Cubana Salud Pública. 2006; 32(4): 0-0.

22. Pineda E, De Alvarado E. Metodología de la Investigación. Organización Panamericana de la Salud; 2008. p. 102.

23. Pilleux M. Competencia comunicativa y análisis de discurso. Estudios Filológicos. 2001; 36: 143-152.

24. Ruiz A, Morillo L. Epidemiología Clínica. Investigación clínica aplicada. Editorial Médica panamericana; 2006. p. 216.

25. Osses S, Sánchez I, Ibañez F. Investigación Cualitativa en Educación. Hacia la Generación de Teoría a través del Proceso Analítico. Estud. Pedagóg. 2006; 32 (1): 119-133.

26. García J, Organista J. Motivación y expectativa para ingresar a la carrera de profesor de educación primaria: un estudio de tres generaciones de estudiantes normalistas mexicanos de primer ingreso. Revista Electrónica de Investigación Educativa. 2006; 8(2): 1-17.

27. Cano M. Motivación y elección de carrera. Rev. Mex. Orient. Educ. 2008; 5(13): 6-9.

28. Avendaño C, González R. Motivos para ingresar a las carreras de Pedagogía de los estudiantes de primer año de la Universidad de Concepción. Estud. pedagog. 2012. 38(2): 21 33.

29. Santos R, Nascimento I. Estudiantes de Psicología mayores de 23 años: Motivaciones y proceso de integración. Rev. bras. orientac. prof. 2012; 13(1):37-47.

30. López M, Hernández C, Polo T et al. Características formativas 
y socioafectivas del alumnado de nuevo ingreso en la Universidad. Revista Electrónica de Investigación Psicoeducativa. 2008; 14(6):95-116.

31. Díaz I, Machado G, Chacón M et al. Rendimiento académico de estudiantes en asignaturas del área básica en policlínicos universitarios. Rev EDUMECENTRO. 2015; 7(1):89-105.

32. Machado B, Llerena M, Mesa L et al. La orientación vocacional: factor determinante en la decisión de estudiar Medicina. Rev EDUMECENTRO. 2013; 5(3): 183-196.

33. Vidal M, Fernández B. Orientación vocacional. Educ Med Super. 2009;23(2):0-0.

34. Maldonado M, Vidal S, Retamal E. Estrategia metodológica para conocer la disciplina como orientación profesional. Educ Med Super. 2011; 25(2):125-134.

35. Peinado F, Fernández M. Reflexión sobre la motivación de los alumnos de grado en la elección de estudios de comunicación en las universidad de Madrid. Estudios sobre el Mensaje Periodístico. 2011; 17(2): 383-400.

36. De la Mano M, Moro M. Motivaciones en la elección de la carrera universitaria: Metas y objetivos de los estudiantes de la Facultad de Traducción y Documentación de la Universidad de Salamanca. Ediciones Universidad de Salamanca. 2013. Pág. 210.

37. Mejías A, Filgueras J, Vázquez M et al. Caracterización de la orientación profesional de estudiantes que aspiran a ingresar a la carrera de medicina. Educ Med Super. 2014; 27(4):374384.

38. Fé de la Mella S, Mirabal J, Contreras J. Factores motivacionales que influyeron en estudiantes de primer año para elegir la carrera de Estomatología. Rev EDUMECENTRO. 2012; 4(3):188-197.

39. Oyarzo C, Vergara M. Reforma de salud en Chile: lo logrado y la pendiente. Disponible en:http://www.expansiva.cl/ media/en_foco/ documentos/12052005115654.pdf Consultado en enero 2016.

40. Verde E, Gallardo G, Compeán S et al. Motivos de elección de carrera en mujeres estudiantes de profesiones de la salud. Educ. med. 2007; 10(1): 44-51.

41. Herrera E, Burgoa T. La importancia del perfil vocacional en la visión del estudiante universitario: caso de estudio en facultades de la Universidad Autónoma de Nueva León, UANL. DAENA: International Journal of Good Conscience. 2012; 8(2): 23-47.

42. Flores M. Razones para el ingreso a la carrera de Odontología. Universidad de los Andes. Venezuela. Revista Odontológica de los Andes 2010; 5(1): 23-30.

43. Sanabria H. Deserción de estudiantes de enfermería en cuatro universidades del Perú. An. Fac. med. 2002; 63(4):301-311.

44. Carrasco E, Zúñiga C, Espinoza J. Elección de carrera en estudiantes de nivel socioeconómico bajo en universidades chilenas altamente selectivas. Revista Calidad en la Educación. 2014; 40(julio):96-128.

\section{Fuentes de financiamiento}

Este artículo ha sido financiado por los autores.

\section{Conflicto de interés}

Los autores declaran no presentar ningún conflicto de interés.

\section{Correspondencia:}

\section{Claudia Troncoso Pantoja}

Dirección: Universidad Católica de la Santísima Concepción.

Facultad de Medicina.

Campus San Andrés. Alonso de Ribera 2850. Concepción. Chile.

Telf.: 56-041-2345427- 56-041-2345406

Correo electrónico: ctroncosop@ucsc.cl

Recibido: 19 de enero de 2016 Aprobado: 17 de febrero de 2016 